\title{
Carcinoma renal de istmo en riñón en herradura. A propósito de un caso.
}

\author{
Andréu García A*, Molina Burgos R*, Coronel Sánchez B*, Navío Perales J**, \\ Botella Almodóvar $R^{*}$, Llamazares Cachá $G^{*}$. \\ *Servicio de Urología. **Servicio de Cirugía General. Hospital Virgen de la Salud de Elda. Alicante
}

Actas Urol Esp. 2008;32(2):249-252

\section{RESUMEN}

CARCINOMA RENAL DE ISTMO EN RIÑÓN EN HERRADURA. A PROPÓSITO DE UN CASO

El riñón en herradura ( RH) es la más común de las anomalías por fusión. La incidencia de carcinoma renal en RH suele ser similar a la del riñón normal. Dadas sus características anatómicas especiales, se deben tener en cuenta para su abordaje quirúrgico, así como para la realización de una posible cirugia conservadora.

Presentamos un caso de carcinoma renal en istmo de RH en el que se realizó cirugía conservadora de ambas unidades renales.

Palabras clave: Riñón en herradura. Carcinoma renal.

\section{ABSTRACT}

\section{HORSESHOES KIDNEY ISTHMUS CARCINOMA. A CASE REPORT}

Horseshoe kidney is the most frequent fusion abnormality of the kidney. The incidence of renal carcinoma in patients with horseshoe kidney is similar to those with normal anatomy. Its special anatomical features must be borne in mind for both surgical approach and conservative surgery.

We present a horseshoe kidney isthmus carcinoma case report in which we performed conservative surgery of both renal units.

Keywords: Horseshoe kidney. Renal carcinoma.

$\mathrm{E}^{\mathrm{t}}$ 1 riñón en herradura $(\mathrm{RH})$ es la más frecuente de las anomalías con fusión renal. Aparece en 1 de cada 400 nacimientos. Más frecuente en varones (2:1), asociándose muchas veces con otras anomalías congénitas. En el 95\% de los casos los dos riñones están unidos por los polos inferiores. EL istmo suele ser de volumen significativo y con parénquima funcionante, se localiza junto a las vértebras L3 o L4 debajo del origen de la arteria mesentérica inferior y suele ser anterior a los grandes vasos, se acompaña de rectificación de los ejes renales y de malrotación de las pelvis renales, los uréteres pueden tener una inserción anormalmente alta.

En un 33\% de los casos son asintomáticos, aunque puede presentar complicaciones en múl- tiples casos, que suelen ser debidas a la uropatía obstructiva y al éstasis urinario (hidronefrosis, litiasis, infecciones, etc.). Además estos pacientes presentan un riesgo de presentar neoplásias, con el desarrollo de carcinomas renales, tumores de Wilms en edad pediátrica y tumores de urotelio ${ }^{1}$.

Por su parte el cáncer de riñón es el tercero en frecuencia dentro de las neoplasias urológicas ( $3 \%$ de los tumores malignos del adulto), siendo la relación 3 a 2 veces más frecuente en el hombre que en la mujer ${ }^{2}$ con un pico de incidencia entre la $5^{\mathrm{a}}$ y $6^{\mathrm{a}}$ década de la vida ${ }^{3}$.

Se trata en el $85 \%$ de los casos, de adenocarcinomas con predominio del subtipo de células claras ${ }^{3}$. 
Desde que en 1895 Hidebrand describió el primer caso de tumor en riñón en herradura, han sido publicados aproximadamente $150 \operatorname{casos}^{4,5}$.

Aproximadamente la mitad de los casos descritos son adenocarcinomas, seguidos por carcinomas de la pelvis renal y tumores de Wilms en proporciones similares cada uno ${ }^{4,5}$.

\section{CASO CLÍNICO}

Paciente varón de 73 años de edad, derivado para estudio y tratamiento de proceso expansivo sólido y heterogéneo, de $10 \times 10.5 \mathrm{~cm}$ de diámetro, sobre línea media abdominal y polo inferior de riñón derecho, en la ecografía abdominal rutinaria.

Había consultado por masa abdominal, dolor y moderada astenia de dos meses de evolución.

Como antecedentes refiere infarto lacunar cerebral con disfasia motora residual, RTU de tumor vesical hace 9 años e hipertensión arterial.

A la exploración se palpa masa dura y móvil en mesogástrio y flanco derecho.

Pruebas complementarias:

La analítica mostró anemia normocítica normocrómica, una función renal dentro de la normalidad y una velocidad de sedimentación elevada.

La ecografia abdominal evidenció proceso expansivo mixto (sólido y quístico) de 10 x 10,5 $\mathrm{cm}$ de diámetro mayor sobre la línea media y polo inferior de riñón derecho.

La TAC confirma los hallazgos ecográficos de masa sólida y quística, de 10 x 10,5 x10 que compromete el istmo en su totalidad sin comprometer a los polos inferiores renales ni a los grandes vasos; tampoco se ve compromiso de suprarrenales, cadenas ganglionares ni vísceras vecinas (Fig. 1).

Se realiza punción aspiración con aguja fina, dando la citología positiva para células malignas con probabilidad de carcinoma de células claras.

Se completo el estadiaje con radiografia de tórax que resulto dentro de la normalidad.

Tratamiento:

Abordaje quirúrgico mediante laparotomía media supra e infraumbilical, decolamiento parietocólico derecho extenso.

Dada la indemnidad renal bilateral y topografia del tumor se decide la resección bilateral del istmo renal y tumorectomía (Fig. 2), previamente se ligaron y seccionaron dos arterias segmentarias.

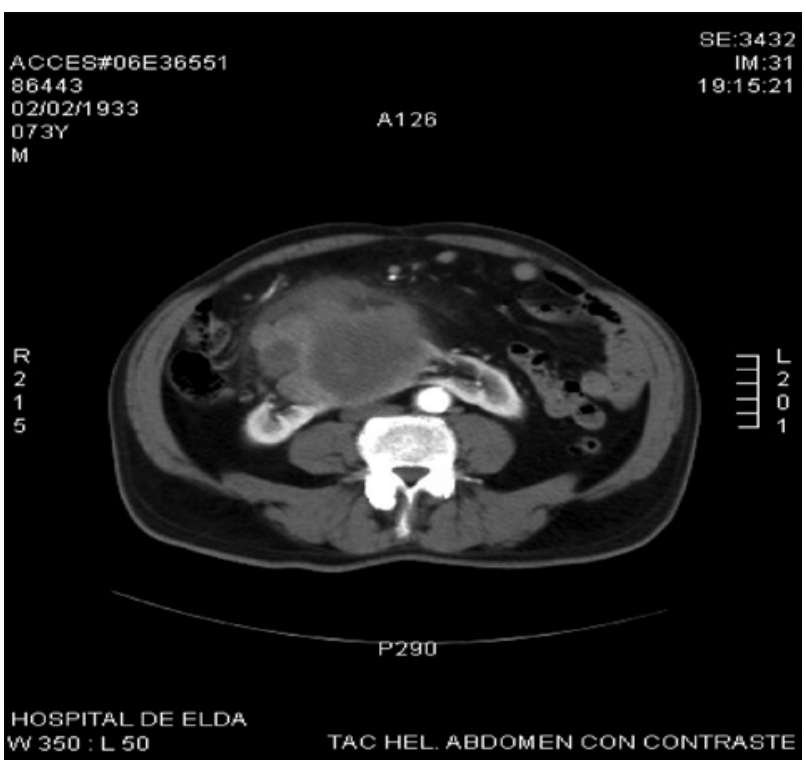

FIGURA 1. TAC Helicoidal abdominal que muestra tumor sólido y quistico de $10,5 \mathrm{~cm}$ de diámetro mayor, localizado sobre el istmo de un riñón en herradura, sin adenopatias ni invasión aparente de tejidos circundantes.

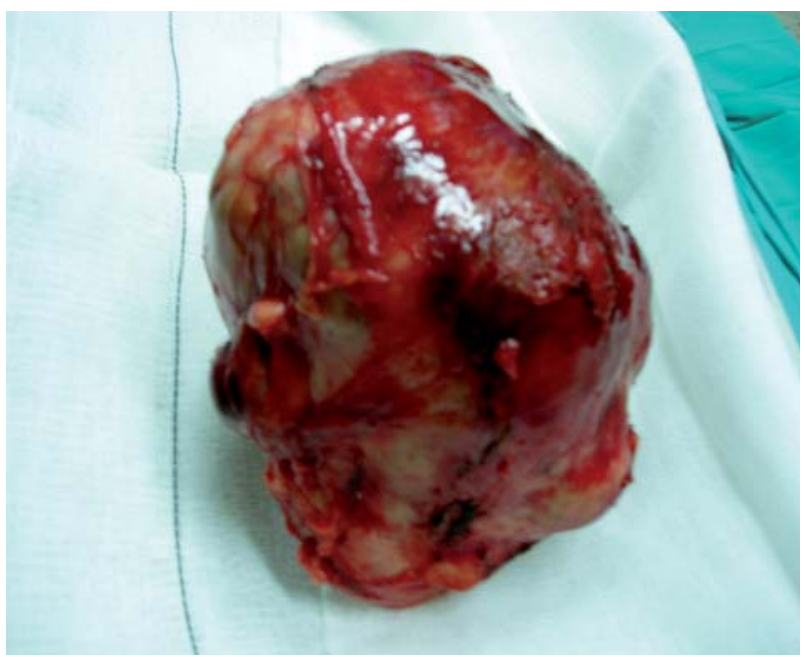

FIGURA 2. Masa tumoral sólida y quistica, con bordes quirúrgicos libres.

Curso postoperatorio sin complicaciones con alta hospitalaria al $7^{\circ}$ día.

La Anatomía Patológica informa de carcinoma renal de 10,5 cm, grado nuclear 2 de Fuhrman, con extensa transformación quistica, sin infiltración de grasa renal, con bordes quirúrgicos libres, ausencia de invasión vascular, ni afectación de ganglios hiliares (Fig. 3).

La neoformación se estadifica como Carcinoma renal quístico de células claras, grado 2 de Fuhrman, T2 N0 Mo. 


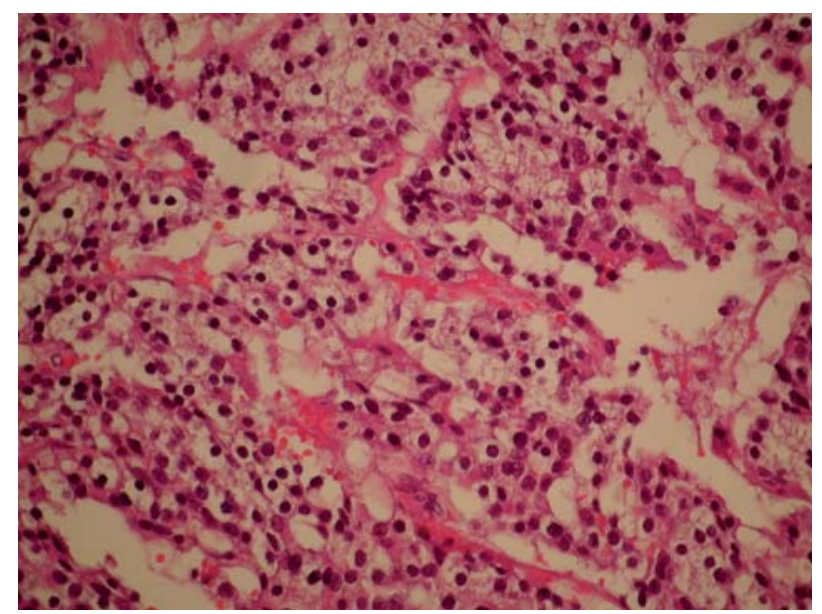

FIGURA 3. Carcinoma renal de células claras, grado nuclear 2 de Fuhrman. Se aprecian células con citoplasma claro y núcleos pequeños e hipercromáticos.

En el seguimiento clínico del paciente a los 6 meses y al año, esta asintomático con función renal normal y TAC helicoidal dentro de la normalidad con hipodensidad en polo inferior de riñón derecho achacable a cambios postquirúrgicos, sin observar adenopatías (Fig. 4).

\section{DISCUSIÓN}

El adenocarcinoma renal es el tipo de tumor más frecuentemente asociado con RH. Puede estar localizado en cualquier lugar del área del $\mathrm{RH}$, incluso 2 de cada 3 afectan al istmo renal ${ }^{6}$. Su pronóstico no está relacionado con su asociación con $\mathrm{RH}$ pero esta determinado por el grado celular, el estadio, el tamaño y el tipo histológi$\mathrm{co}^{5,7}$.

Actualmente realizar una $\mathrm{TAC}^{8,9}$ se considera esencial en el protocolo diagnóstico y se recomienda llevar a cabo una arteriografia antes de la cirugía dada la presencia de anomalías vasculares en aproximadamente en el $70 \%$ de los casos y la frecuente asociación con hematomas y abscesos perirrenales ${ }^{6}$.

Sin embargo actualmente se sugiere el empleo de la TAC Helicoidal con reconstrucción en tres dimensiones como único estudio, dado el detalle anatómico de alta resolución que ofrece la prueba ${ }^{8}$.

La técnica quirúrgica que ofrece el mejor acceso al RH es la laparotomía media, sobretodo por la disponibilidad para el control vascular temprano de los grandes vasos y hemostasia selectiva de arterias del istmo renal $6,9,10$.

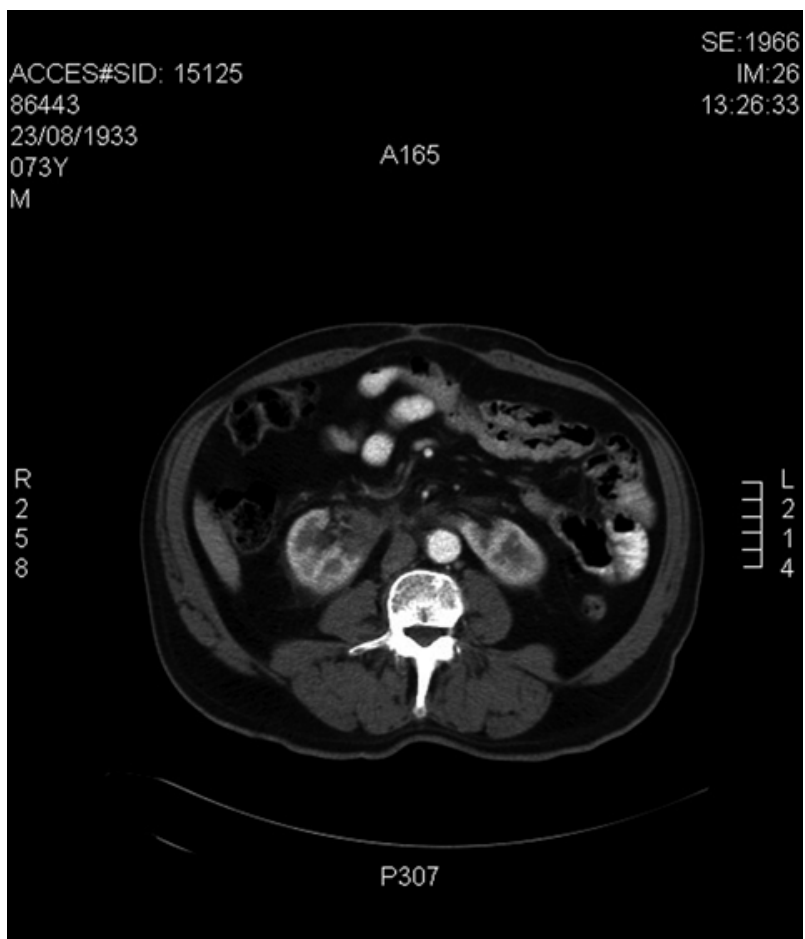

FIGURA 4. TAC Helicoidal abdominal con área de hipodensidad en polo inferior de riñón derecho achacable a cambios postquirúrgicos, no se objetivan masas dependientes de riñones ni adenopatias.

Cuando se plantea la intervención, hay que considerar el posible crecimiento del tumor a través de un istmo parenquimatoso, como es el caso que nos ocupa, lo que puede hacer necesaria la ectomía de las dos unidades renales, o si el tumor está confinado al istmo, realizar la istmectomía y tumorectomía si es factible ${ }^{6}$.

\section{CONCLUSIÓN}

El RH es la más común de las anomalías por fusión, con un predominio en el sexo masculino.

Casi un tercio de los portadores de esta malformación congénita permanecen asintomáticos , cuando se manifiestan clínicamente lo hacen a causa de hidronefrosis, litiasis, infecciones y con menor frecuencia tumores.

El carcinoma renal es el tipo de tumor más frecuentemente asociado con RH. Su incidencia es similar a riñones normales. Su pronóstico depende del grado y del estadio local, como en los riñones normales. La cirugía conservadora de nefronas debe tenerse en cuenta para el carcinoma renal en $\mathrm{RH}$, sobre todo para los tumores detectados incidentalmente. 


\section{REFERENCIAS}

1. Bauer SB. Anomalias del tracto urinario superior, "Campbell Urología” $8^{\mathrm{a}}$ edición. Ed. Panamericana - Buenos Aires, 2004; 2080-2084

2. Landis SH, Murray T, Bolden S, Wingo PA. Cancer stadistics, 1999. CA Cancer J Clinic. 1999;49(1):8-31.

3. Ortiz Hering FL, Beneduzzi Neto L.: Câncer do Rim. Urologia-Diagnóstico e Tratamento: 377-382, Platina, Sâo Paulo, 1998 .

4. Torrubia Torrubia Romero FJ, Murillo Mirat J, Caballero Gómez M, Asuar Aydillo S. Adenocarcinoma renal en el istmo de un riñón en herradura. Arch Esp Urol. 1993;46(1): 43-46.

4. Torrubia Torrubia Romero FJ, Murillo Mirat J, Caballero Gómez M, Asuar Aydillo SRenal adenocarcinoma in the isthmus of a horseshoe kidney. Arch Esp Urol. 1993;46(1): 43-46.

5. Vázquez Blanc S, Calahorra Fernández L, Rodríguez Antolín A, García Luzón A, Carrero López V, Pamplona Casamayor $\mathrm{M}$, et al. Patología tumoral en el riñón en herradura. Actas Urol Esp. 1994;18(7):764-767.

5. Vázquez Blanc S, Calahorra Fernández L, Rodríguez Antolín A, García Luzón A, Carrero López V, Pamplona Casamayor $\mathrm{M}$, et al. Tumor pathology in horseshoe kidney. Actas Urol Esp. 1994;18(7):764-767.
6. Rubio Briones J, Regalado Pareja R, Sánchez Martín F, Gilberto Chéchile T, Huguet Perez J, Villavicencio Mavrich H. Eur Urol, VI,4: 306-310, 1999.

7. Algaba F, Arce Y, Trias I, Santaularia JM, Antonio Rosales A. Aplicación clínica de las actuales clasificaciones del cáncer renal. Actas Urol Esp. 2006;30(4):372-385.

8. Lee CT, Hilton S, Russo P. Renal mass within a horseshoe kidney: preoperative evaluation with three-dimensional helical computed tomography. Urology. 2001;57(1):168.

9. Otero-García JM, Maldonado-Alcaraz E, López-Sámano VA. Carcinoma de células claras en riñón en herradura. Descripción de un caso y revisión de la literatura. Gac Méd Méx. 2005;141(4):305-307.

10. Elias R, Rico D, Rodríguez R, Viale S. Adenocarcinoma renal en el istmo de un riñón en herradura. Rev Arg Urol. 2004;69(4):248-251.

Correspondencia autor: Dr. A. Andréu García Servicio de Urologia. Hospital Virgen de la Salud Carretera de la Torreta s/n - 03600 Elda (Alicante) Tel.: 966989000

E-mail autor: thader02@hotmail.com

Información artículo: Nota clínica Trabajo recibido: noviembre 2006

Trabajo aceptado: diciembre 2006 